\title{
Competences for Digital Transformation: Insights from the Norwegian Energy Sector
}

\author{
Karen S. Osmundsen \\ Norwegian School of Economics (NHH) \\ karen.osmundsen@nhh.no
}

\begin{abstract}
Numerous established firms are undergoing digital transformations. To manage a digital transformation (DT), companies should develop DT capabilities. One important prerequisite for DT capabilities is DT competence - a bundle of employee skills, knowledge, and expertise necessary for a successful DT. However, research on which competences are essential for a DT is limited, as is research on how established firms can obtain these competences. Based on a longitudinal case study, this paper provides insight into which competences were identified as important for the DT at an established firm in the Norwegian energy sector, and how these competences were obtained. The paper also presents a conceptual model, to better understand and study the concept of DT competence as a prerequisite for DT capabilities.
\end{abstract}

\section{Introduction}

The term digital transformation (DT) is increasingly applied to describe the major changes organizations are undergoing in the digital era. DTs are motivated and incentivized by emergent digital technology, changes in the competitive landscape, and increased customer centricity [19], and imply organizations partly or completely altering the way they do business. For many organizations, a DT is necessary to stay relevant and competitive, and to retain leadership positions [22]. This is true especially for established companies. As Sebastian and colleagues [22] nicely put it: "Since past success does not ensure future success, older companies will need to transform to take advantage of digital era opportunities" (p. 206).

DTs occur in private and public companies in almost every industry, including the energy sector [14]. Enabled by digital technology, the entire system for producing and distributing energy is changing, and trends such as electrification, self-generation, and local energy solutions (micro-grids) are being introduced. Moreover, an increasing number of digital elements and smart components, such as automated meter reading (AMR) in Norway, are incorporated in all layers of the energy network [14]. With AMR technology, grid companies are able to collect accurate and nearly real-time data for energy consumption. AMR data, in combination with other available data, provide grid companies with a richer and more accurate foundation for improving services and accommodating customers.

At the same time, new competitors, ranging from larger enterprises and non-industry entrants to smaller startup companies, are entering the energy sector. Additionally, in the Norwegian context in particular, regulatory actors are introducing regulations and guidelines that may change the dynamics in the industry, leading the players toward cooperation, changed roles, new business models, and new ways to approach end users. An example in Norway is the newly established Elhub, which has altered the roles in the Norwegian energy sector. The Elhub is a central data hub for handling meter readings and market processes in the Norwegian energy sector. The hub was established in February 2019 , to improve the efficiency of the distribution of meter readings, by providing high-quality data and leveraging the digital potential derived from AMR.

The digital trends and development in the energy sector are transforming grid networks into so-called smart grids: "intelligent networks with digital processing and communication enabling continuous data flow and information management control" [14, p. 3]. Smart grids can be seen as power grid networks coupled with information systems and digital technology. Grid companies face the need to digitally transform to leverage the digital development and prepare the organization for future smart grids.

Researchers have suggested several elements that should be in place for organizations to succeed with DTs, such as a supportive organizational culture, well-managed transformation activities, a digital business strategy, and alignment of business and information systems (IS). Further, organizations should leverage external and internal knowledge, engage managers and employees, develop dynamic capabilities, and grow IS capabilities [19]. 
IS capabilities are meta-level constructs that are underpinned by different competences embedded in organizational processes, and rooted in employee skills and knowledge [20]. Therefore, a DT often requires a transformation of the workforce [9], and is associated with, among other structural changes, changes in employee roles and skills [25]. To increase IS capabilities, or digital capabilities, organizations should build their own digital talentseither by training internal resources or by acquiring external resources $[3 ; 10]$. For established firms, this strategy implies taking advantage of existing strengths and resources, and at the same time, developing digital skills and exploiting new digital technology [20]. However, insight is limited into how established firms can accomplish this strategy [9], and which competences and skills are particularly important for DTs [6;23].

Drawing on resource-based theory (RBT) and DT literature, the aim of this paper is to investigate which competences are important for established firms to succeed with DTs. Based on a longitudinal case study, I examined which competences were essential for an established firm in the Norwegian energy sector in managing and embracing their DT, and how these competences were obtained in the organization. The following research question is addressed: Which competences are essential for DTs in established firms, and how can these competences be obtained?

This paper precedes as follows. First, relevant literature on RBT and DT is presented in sections 2 and 3. The research method is described briefly in section 4 , and then the case is presented in section 5 . The findings are presented in section 6 , which are further discussed in section 7 . The paper is concluded in section 8, and limitations and future research areas are discussed.

\section{RBT and capabilities for DT}

According to RBT, firms are different from one another because of their resources. At the core of RBT is the idea that resources, skills, and capabilities that are firm specific, rare, and difficult to imitate by rival firms can give rise to competitive advantage and superior organizational performance [4;5]. Drawing on this idea, research on RBT often focuses on how companies can exploit existing firm-specific resources, and at the same time, expand their strategies for developing new resources, skills, and talents [24].

Although rooted in management research, RBT and capability approaches have been applied extensively in the IS literature to explain how organizations gain superior performance $[5 ; 18 ; 26]$.
There is consensus that technology itself has little value, and that IS alone is unlikely to create sustainable competitive advantage [20]. However, researchers have identified IS capabilities as key organizational capabilities that can contribute to sustainable competitive advantage if the following characteristics are found: rarity, non-substitutability, and non-replicability $[5 ; 18 ; 26]$.

IS capability can be seen as a company's ability to assemble and deploy IS-based resources in combination with other firm-specific resources [5; $18 ; 26]$. IS capability represents a company's ability to connect technology to the company's business [20], and to leverage technology to differentiate the firm from its competition [5; 20]. More recently, the term digital capability has emerged in the literature, and is described as a fundamental building block for a successful DT. Despite the common use of the terminology, there are different views on what digital capabilities actually imply, and how they contribute to a successful DT. One perspective is that digital capabilities are a firm's skills, talent, and expertise in managing digital technologies [15]. Further, some researchers instead use the terminology digital transformation capabilities, to denote the capabilities necessary for DT. Following Wiesböck and Hess [27], digital transformation capabilities can be seen as an "organization's ability to implement digital business concepts that complement digital solutions (i.e., handle the process of digital transformation)" (p. 10).

When the literature on the capabilities and competences essential for DTs is explored, it becomes apparent that the terminology is used in different ways, and with different meanings, by different researchers. In particular, the distinction between capability and competence is confusing in the literature [20]. For the purpose of this paper, and to ensure the terminology I use is clearly understood, in structuring the concepts, I draw inspiration from Peppard and Ward's [20] IS capability model (see Figure 1).

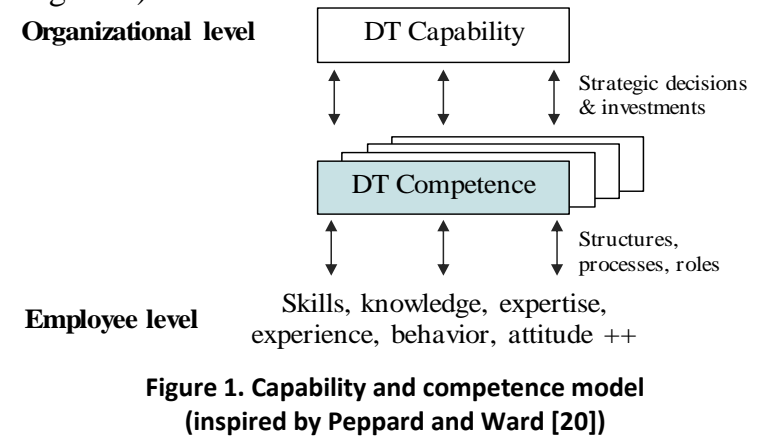


Based on Figure 1, DT capability, a firm's ability to manage a DT, is a construct at the organizational level. To achieve DT capability, a firm must make well-thought-out investments and strategic decisions related to areas such as digital technology, infrastructure, leadership, culture, and competence. DT competence is an important prerequisite for DT capability, and represents a bundle of employee's competences that are essential for DT. Skills, knowledge, expertise, and other attributes of employees across the organization are managed through structures, processes, and roles, to reflect the necessary competence base for DT.

\section{Competences for DT}

DT implies leveraging and integrating new digital technologies in business processes, to enable major business improvements [21]. However, as new digital technologies are introduced in a firm, new skillsets are required $[6 ; 25]$. Thus, the firm's human capital and competence management are important for a successful DT. In this paper, I define DT competence as a firm's bundle of its collective competences (skills, knowledge, expertise, experience, and other employee attributes) that are essential for a DT, and includes the firm's ability to combine these attributes in responding to and managing the DT. According to Butschan et al. [6], empirical evidence for DT competence is limited. To reduce this gap, the authors developed and tested a competence model for DTs, and found that cognitive competences are particularly important for a successful DT. In their study, cognitive competences include willingness to learn, openness to change, problem-solving capability, and expertise [6].

The expertise dimension presented by Butschan et al. [6] is linked to specific digital technologies, and which expertise is relevant varies from one company to another [13]. Digital trends in the sector in which the company operates also play a role. In the energy sector, researchers recommend that the growing importance of smart digital solutions should be taken into account when preparing the workforce for future scenarios [14]. As future power grid networks are likely to involve more "software and services, platforms, interconnections, and system optimization of the increasingly complex systems" [14, p.3], acquiring and developing expertise on relevant digital technologies is regarded as essential in the DT of grid companies. Examples of the relevant expertise found to be important for DT include (big) data analytics [8; $13 ; 15]$, computer science [14], Internet of Things [6], social media, mobile [15], robotic process automation, and cloud computing [3].
Besides cognitive competences, Butschan et al. [6] found that discipline, ability to execute, ability to organize, and conscientiousness (what the authors termed processual competences) are important competences for DTs. Andriole [3] highlighted competences within business process management (BPM), agile program management, cybersecurity, and effective communication skills. In contrast, Butschan et al. [6] found that communicative ability, together with other social competences, has only a limited effect on the success of a DT. The authors suggested this might be the case as DTs are mostly driven by technology, and thus, social competences are less useful.

As important competences for DT are identified, companies should assess the inherent competences represented by the existing workforce, and react accordingly if gaps exist [3]. Companies, in general, have three options for reducing competence gaps: (1) develop competences internally, (2) acquire relevant competences externally, or (3) rent competences from the environment. A combination of these approaches is often appropriate.

The first option implies that companies build DT competences internally, mainly through offering employees relevant training and education $[8 ; 15]$. Training could include specific and general training levels, and should be supported by skill-building units [15]. Moreover, companies should ensure that employees have access to relevant technology and resources supporting the technology and its application [16], and nurture a digital culture that encourages and motivates learning and interest in digital technology.

The second option involves recruiting the relevant DT competences externally. According to Kettunen and Mäkitalo [14], several companies in the energy sector already specify DT-relevant skills and specifically, digital technology expertise, as requirements in their job recruitment descriptions. In Norway, and in other countries, digital technology expertise is a scarce resource [11], and companies need to put substantial effort into attracting the right employees. This could be done through creating and stating an explicit digital profile, fostering and encouraging a digital culture, and offering exciting and challenging opportunities and tasks to future employees.

The third option involves engaging with specialized consultancies [8], contractors, or longterm vendors [3], and entering into collaborations or alliances with strong players in the environment [15]. In the energy sector, grid companies are investing in research and development (R\&D) projects in collaboration with strong digital players and other 
companies. Such collaborative efforts contribute to learning across company borders, and in developing DT competences [14].

\section{Research method}

Insight to answer the research question was derived from a case study at an established firm in Norway. A case study involves studying a case within a real-life, contemporary context over time, through detailed, in-depth data collection from multiple sources of information [7]. The main source of data was interviews. I conducted semi-structured interviews with 44 participants in different positions and departments in the company, in addition to several informal meetings and conversations. Each interview lasted between 30 minutes and 2 hours, and was recorded, transcribed, and coded. Because of space limitations, a full list of interviewees is not included in this paper. However, an overview of the interviewees, their length of experience at the company, and their highest education level is presented in Table 1.

Table 1. Overview of interviewees

\begin{tabular}{|c|c|c|c|c|c|c|}
\hline $\begin{array}{c}\text { Education } \\
\text { / Years at } \\
\text { GridCo }\end{array}$ & Apprentice & $\begin{array}{c}\text { BSc/ } \\
\text { MSc } \\
\text { electro }\end{array}$ & $\begin{array}{c}\text { BSc/ MSc } \\
\text { IT }\end{array}$ & $\begin{array}{c}\text { BSc/ MSc } \\
\text { other }\end{array}$ & $\begin{array}{c}\text { Technical } \\
\text { college }\end{array}$ & Total \\
\hline $0-5$ & & 2 & 2 & 4 & & 8 \\
\hline $5-10$ & 2 & 4 & & 2 & & 8 \\
\hline $10-20$ & 3 & 2 & & 4 & 2 & 11 \\
\hline $20-30$ & & 1 & & 2 & & 3 \\
\hline $30-40$ & 6 & 3 & & 3 & & 12 \\
\hline $40-50$ & 1 & & & & 1 & 2 \\
\hline Total & 12 & 12 & 2 & 15 & 3 & 44 \\
\hline
\end{tabular}

The research was longitudinal, as I followed the case organization over a long period. To this point, I spent 2-3 work days per week with the company over a 9-month period. Such access to the organization, enabled me to conduct rich observations, and reveal issues and view the organization from an insider perspective [12]. Observations were documented in field notes, and constitute a valuable data source and a foundation for data analysis and further data collection. Further, I have had access to extensive amounts of internal and external documents, as a supplement to other data sources, providing rich insight into the case organization and their DT.

\section{The case: DT at GridCo}

The case organization, referred to as GridCo, is a grid company in Norway, and a large player in the Norwegian energy sector. Similar to other grid companies in Norway, GridCo is responsible for building, operating, and maintaining the regional and local distribution network in a specific geographic area, and thus, for providing electricity to customers.

Founded in the early 1900s, with approximately 400 employees and long traditions in serving their obligation to provide electricity to customers, GridCo can be considered an established firm. Further, GridCo is in the middle of a DT, dealing with the aforementioned trends and development in the energy sector, and thus, is a relevant case organization for this research. The company has ambitious strategic goals and visions for the future, with plans of great growth and expansion. For GridCo, these plans imply major changes to the entire organization, the ways of working, and possibly even the business model.

In 2014, the company engaged in an enterprisewide project labeled The Future Grid Company, whose goal was to analyze and establish efforts for the company to improve profitability, better leverage digital technology, and meet the demands and digital trends in the environment. Although previous initiatives and events also played a role, in one sense, this marked the beginning of the DT of GridCo. Figure 2 illustrates some of the events and initiatives that have shaped the DT of the company since then, in particular in relation to the competence perspective.

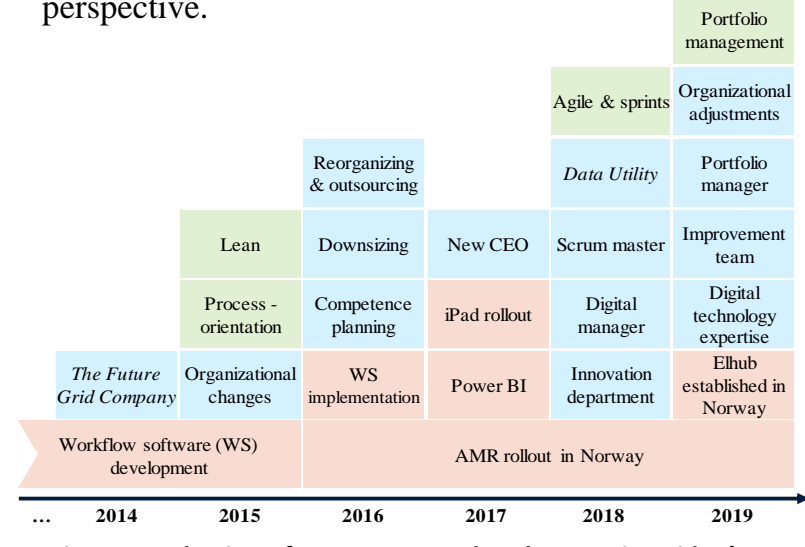

Figure 2. Selection of competence-related events in GridCo's DT

The blue boxes in Figure 2 reflect events directly associated with the company's development of competences, including changes in roles and the organizational structure. The green boxes represent events associated with methodology and ways of working, whereas the orange boxes represent digital technology-related initiatives. However, not all events that may have had an effect on the company in its DT are presented here. The events presented in Figure 2 are those that the data revealed as most relevant from a competence perspective. In addition, the company is experimenting with and investing in 
several emerging digital technologies; however, for the purpose of this paper, only some are discussed.

The Future Grid Company project led to a number of initiatives to better prepare the company for the future, such as introducing a process-oriented mindset and Lean as a philosophy of working. To become more efficient and to gain a better advantage from digital technology and digitalization, the company saw the need to restructure the entire organization. As a result, 2016 was a year characterized by reorganization, outsourcing of services, and large-scale downsizing. To support these efforts, GridCo established a routine for competence planning. Twice a year, the management at GridCo work on adjusting the company's strategic competence plan, which is nested in the entire organization. The intention of the competence plan is to investigate how the company can ensure the right competence to accommodate existing and future business in the short and medium term.

During 2016, a self-developed software for managing the entire workflow of the company's core business was also implemented. The software supports employees and processes associated with projecting, planning, executing, administering, and following up with building new, and maintaining existing, parts of the power grid. Introducing the software implied introducing a new way of working for the majority of the workforce at GridCo.

One group of employees especially affected by the events in 2016 were the electricians. Electricians make up almost half of the GridCo workforce, and are the employees who execute the company's core business: ensuring electricity is provided to new and existing customers. Implementing the software eventually involved equipping each electrician with an iPad. The iPad, with the software installed, was intended as a tool for the electricians in receiving work orders with relevant information, managing their workday, checking off executed work orders, and documenting their work. The executing part of the electricians' job is not affected by the software or use of iPad. One of the interviewees commented, "you can talk about digitalization and robotization, and the like, but our grid system is how it is. Some of it was built over 100 years ago, and still exists. So, we need to be there physically to execute the work (...). Someone still has to do the physical job-we can't escape that" (Interviewee 7). Nevertheless, replacing sticky notes and slow computers with iPads, and introducing a software and structure for managing work orders, was expected to have a big impact on the time spent by electricians before and after they physically execute a work order.
One aspect, however, did not receive adequate attention in the implementation phase: The majority of the electricians had limited experience with using an iPad, and even less with using mobile applications, such as the workflow software. Thus, the introduction of the new software, and the iPad as a tool, required the electricians to develop new skills, and master new types of digital technology.

In 2018, inspired by the newly appointed chief executive officer (CEO), a separate innovation department was established at GridCo. The department was founded to manage and oversee processes related to innovation, strategy development, R\&D and improvement projects, and digitalization, among others. Then, a digital manager was appointed internally to oversee digital initiatives and digitalization efforts, in collaboration with the chief digital officer (CDO) of the parent company and the digital managers of other subsidiaries. This role was created to ensure that the company group had a collective viewpoint on the DT, could exchange experiences and ideas, and would benefit from digitalization efforts across the subsidiaries.

During the same year, the increasing use of digital technology in the company and the implementation of AMR across the country encouraged GridCo to develop a strategy A3 labeled Data Utility. A strategy A3 is a problem-solving tool from the Lean toolbox, which helps companies in stating the current situation and the problem to solve, highlighting goals, proposing and planning appropriate measures, and following up and ensuring targets are reached. The Data Utility A3 incorporated several aspects of digital business development, and how to utilize increased amounts of data. The competence perspective is a major part of the A3. In collaboration with the work on competence planning, one main contribution from the A3 was identifying the competence necessary to leverage and benefit from emergent digital technology and increasing amounts of data. Competence gaps were mapped based on future needs and existing competences in the company, and a plan was created to ensure the necessary competence.

\section{DT competence at GridCo}

The work on strategic competence planning and the strategy A3 collectively provided GridCo with relevant insight into the company's current competence base, as well as the competence needed for the company to better embrace the DT. Based on this information, before taking any measures, the company thoroughly considered whether anything could be done to better mobilize the current 
competence base. This evaluation involved assessing whether competence could be better utilized through changes in tasks, responsibilities, organizational structure, motivational measures, management, redeployments, or the like. All levels of the organization were evaluated before any measures were taken. After the mobilization, two main approaches for ensuring DT competence were deployed at GridCo: (1) developing DT competence and (2) acquiring DT competence.

\subsection{Developing DT competence}

The first approach involved GridCo facilitating skill development and knowledge sharing through supporting continuing education, offering relevant courses and training, and encouraging self- and peer learning. Employees also participate in digitalization projects in the company, and are offered opportunities to attend external seminars to get inspiration, and learn from others.

As an example, as the new workflow software and the use of smart devices in relation to work were new to the majority of electricians, the company offered training to develop digital technology orientation and digital technology adoption among electricians. Digital technology orientation refers to not only the willingness and capacity to use digital technology but also the general awareness of and interest in digital technology that can lead to a digitally enabled work-life [1]. Digital technology adoption refers to the application of digital technology, and occurs when employees realize or decide that the digital technology has utility, and can add value to their work and tasks [1].

However, as pointed out by several interviewees, the first training offerings were not well received by employees. Large groups, general content, and no individual customization led to frustration and limited learning. One interviewee commented, "They gathered a lot of people, and then they rushed through it. The electricians were present, but they could have talked about whatever, and they wouldn't have remembered anything anyway (...) Training is crucial. You have to get people on board, even those who resist. It's mostly just about them not understanding [the technology], and being insecure in how to use it" (Interviewee 10). After receiving feedback from users, the company improved the training. GridCo started offering more thorough and customized training sessions, in smaller groups, to meet the different needs and demands of the electricians. Further, as one interviewee commented, "tutoring videos are now published regularly to help explain specific issues requested by users" (Interviewee 5).

GridCo also offers training and introductory courses to increase orientation to and adoption of other digital solutions, such as the business intelligence (BI) tool Power BI, drones, and virtual reality (VR). Regarding the last, the company arranged a VR day in June 2019, where employees could learn more about VR, and how it can be applied to improve their work tasks, and even test the technology in relevant surroundings. Some employees have also taken the initiative to find and attend relevant external courses, such as about big data, which the company has supported.

GridCo also identified analytical skills as necessary skills that could be developed internally. In particular, the company saw a need to develop analytical skills to better measure and monitor performance, and to be able to make better, and datadriven, decisions. A manager pointed out: "measuring and planning have been based on guesswork, and not on actual data. It's like navigating based on the track of the ship-you look over your shoulder to see how you managed" (Interviewee 13). In 2017, Power BI as a tool was acknowledged as a good starting point, and two employees who showed specific interest encouraged to allocate some of their work day to learn the tool. These employees had no formal IT technical background, but a higher-than-average curiosity and interest in digital technology, and how it can be applied to improve their work. After a while, the employees mastered the tool, and developed analyses and reports on request for managers across the entire company. This development was highly appreciated by managers at different levels. One stated, "What has changed the last few years is that data is much more available. Previously, we made decisions based on a 'picture' we had-we had a perception about something, or we had to spend a lot of time to find the data (...) Now data is much more available, and we use Power BI actively in decision-making" (Interviewee 1).

The Power BI example above is an example of GridCo's encouragement of self-learning. The company also realizes they have a lot to learn from other companies, as well as from each other. Traditionally, mainly managers attend external seminars and conferences to get insight into the possibilities for using digital technology to improve processes, products, and services. More recently, however, the company has acknowledged the benefits of having other employees, and not only managers, attend such events. For instance, in 2018, five employees from different areas of the company 
attended a conference in London on grid analytics, industry-specific analytics. There, the employees were exposed to the latest digital innovations and solutions within the energy sector, and within grid networks in particular. The employees, who work in the grid network on a daily basis, came back to the company, glowing and inspired, and with plenty of ideas for how GridCo could use digital technology to improve services. After the employees returned, they shared their thoughts and ideas with management and other groups of employees at the company, on several occasions. This is an example of learning from external others, and learning from each other. Further, letting employees, rather than managers, have this kind of experience sends a positive signal to all employees, and helps in building a digital culture.

GridCo has also focused on process improvement and digitalization efforts in their DT. The newly developed workflow system represents both. The development and implementation of the system digitalized and increased the efficiency of the processes for building and maintaining the power grid, from planning the work to documenting the finished work. The system further represents a bottom-up approach, where a group of employees initiated the development. They saw the need for a system that accommodated entire processes, integrated the different IT systems involved, and created more transparency in the workflow. Employees from the business units, not only the IT department, were involved in developing and implementing the system. They are still involved, as the system is constantly evolving, based on feedback and ideas from different users, whether they are electricians, work planners, project engineers, managers, or administrative staff.

\subsection{Acquiring DT competence}

The second approach involved GridCo hiring resources with relevant and necessary skills. Positions are first announced internally in the company, followed by external job advertisements if the right candidate is not found in-house. This typically involves recruiting persons with knowledge and expertise that is new and unfamiliar to the firm.

GridCo has identified a need to recruit persons with specific digital technology expertise in robotization, machine learning, sensor technology, and big data, among other areas. As an example, in January 2019, the company hired two data scientists. Their main job consists of applying their expertise in programming, machine learning, and data analysis, to find ways to better utilize the massive amounts of data that are available to the company. This job is especially important and valuable, as the AMRs and Elhub provide the company with increased amounts of real-time data. Further, GridCo has recruited summer interns who, during the summer of 2019, are working with different types of digital technology and data analysis, including analyzing data from drones and experimenting with image recognition and machine learning.

In addition, to further build on the Power BI work in the company, GridCo decided to strengthen the analytical skills even more, and hired a BI developer in April 2019. The company reasoned that the Power BI work had become an important tool for measuring performances; however, the work lacked structure, and had limited scalability. Thus, the company acquired external skills in BI development, to improve business intelligence in the company: "to build more specialized competence, to be able to use the data in a better way, to visualize data better, and to have a bit more control" (Interviewee 30).

Despite acquiring competence with digital technology expertise and analytical skills, GridCo still acknowledged having limited knowledge of the data available. Therefore, the company decided to acquire competence in data management. One person is recruited, starting September 2019, with the preliminary job title master data management (MDM) advisor. The main tasks of this role is to structure the way data are gathered, stored, and used, and take necessary measures to increase data completeness and quality across the organization.

GridCo is engaged in numerous digitalization initiatives, from small day-to-day improvements to large $R \& D$ projects in collaboration with external parties. However, as the number of such initiatives increased, management felt they were losing control over the many efforts across the company. Thus, the company decided to improve the structure and process of improvement and $R \& D$ projects in the company through portfolio management. To achieve this, a portfolio manager was recruited in January 2019. This employee has built a structure for prioritizing projects, based on criteria such as feasibility, strategic fit, risk, and cost. Company policy now states that no project (over a certain cost limit) is to be initiated without passing through the portfolio funnel. The portfolio manager, together with the newly established improvement team, is also working on establishing channels for employees to suggest areas for improvement and digitalization.

At the same time, digitalization efforts and projects at GridCo often moved slowly. To deal with this slow pace, agile project management was introduced as a methodology, following the recruitment of a Scrum master in January 2019. The 
Scrum master introduced sprints, a concept from agile project management, where certain tasks or activities are to be completed within a set period. Sprints were introduced to enable and accelerate change initiatives in the company, "through focused and systematic work in dedicated and multidisciplinary teams" (personal communication, 20.05.2019). In Figure 3, the different skills and employee attributes that make up the DT competence in GridCo are summarized

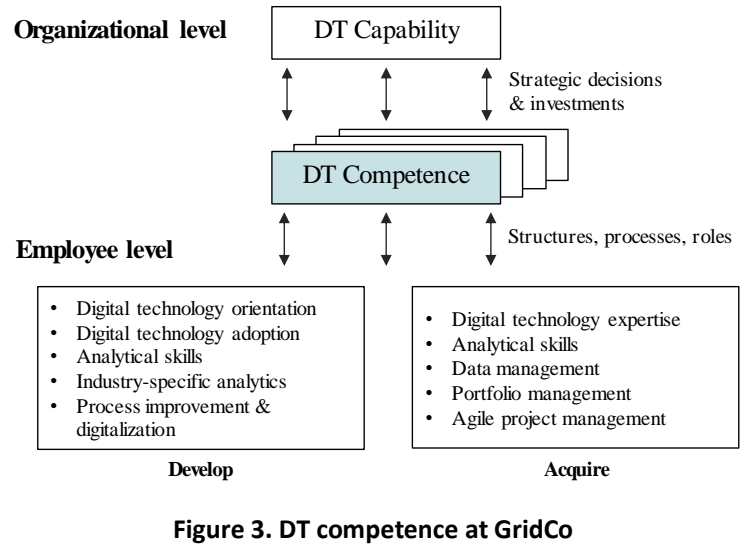

During the last few years, GridCo has engaged in what they refer to as a competence shift in the company, where "the competence base within electrical power engineering is being supplemented with competence within digital technology" (personal communication, 14.03.2019). This type of competence shift is expected to continue to underlie the competence planning in the near future, affecting the entire organization.

\section{Discussion and implications}

The competences found as essential in GridCo's DT, to some extent resemble the competences pointed out by other researchers as important in DTs. The cognitive competences Butschan et al. [6] identified, willingness to learn, openness to change, and expertise, are similar to digital technology orientation, digital technology adoption, and digital technology expertise in this study. Some lines can also be drawn from process improvement and digitalization identified in this study to Butshcan et al.'s [6] problem-solving capability. Similar to prevailing research $[8 ; 13 ; 15]$, I also found analytical skills as especially important for managing a DT. I found industry-specific analytics, in this case, grid analytics, as essential for GridCo's DT, which is in line with the recommendations of Kettunen and Mäkitalo [14]. In conformity with Andriole [3], I also found agile project management to be important for the DT at GridCo. Together with portfolio management, these skill areas resemble the elements of what Butschan et al. [6] termed processual competences, although the competences and skills identified in this study were not as specific. Competence within data management, however, does not seem to have received much attention in literature. This may be because this skill is considered an integrated part of data analysis, and thus, is not viewed as a separate skill or competence. At GridCo, however, data management is seen as a particularly crucial prerequisite for data analysis, to cope with the increasing amounts of data. Thus, data management is presented as a separate skill necessary for the company in their DT.

A DT is a continuous process, without necessarily any clear beginning and end. The findings in this study represent competences that at GridCo appear to be necessary for their DT up to this point and in the near future. The company will continue with their approach to competence planning. As new digital solutions arise, and employees become more familiar with digital technologies, GridCo will likely identify new competence gaps. One manager, for instance, contended that in the future GridCo may have to alter the apprentice arrangement for electricians, adding more subjects within digital technology: "There will be much more 'thingies' to assemble in the future. Raising a pole is really rather rough, mechanic work. But if that pole is equipped with a sensor or the like, the electrician will have to know something about sensor technology and how the sensors communicate - to be sure that he or she assembles it correctly" (Interviewee 13). Further, development of the energy sector, and changes in the current business model, is likely to lead to new service offerings and tasks, which will affect the competence needs of the company to continue embracing the DT.

In addition to the findings on DT competences at GridCo, and how the company obtained these competences, there are three important implications to draw from this study. First, before taking any measures to reduce competence gaps through development or acquisition, companies should evaluate, and consider possibilities for mobilizing the current competence base. Perhaps an employee enjoys programming as a hobby, and his or her skills could be better utilized if he or she was given other tasks or organized in a different department. Such insight requires thorough mapping and understanding of the current competence base, beyond merely the level of education and previous work history. It requires listening to employees to uncover their undocumented skills, interests, experiences, attitudes, etc. At GridCo, this need is met through individual 
development plans, as a result of continuous dialogue between employees and their managers. To support this process, the company uses a specialized tool for competence management and training overview, where employees and managers can manage relevant training for the employees to meet requirements or individual goals.

Second, mobilizing, developing, and acquiring competence will change the competence base of the company, and may require some restructuring of the organization. According to Dremel et al. [8], an important stage in a DT involves leveraging the newly added competence in the company. One approach is to make organizational adjustments, adapted to the digital age, to better accommodate the new competence base. This could involve developing competence centers, ensuring clearly defined roles and responsibilities, and aligning business and IT [8]. At GridCo, the establishment of the innovation department represents such an effort, to build up expertise and skills in an organized way. Adding roles such as digital manager, Scrum master, and portfolio manager also reflect measures the company has taken to oversee, organize, and approach initiatives underlying the DT. Further, GridCo is currently working on adjusting its organizational structure again (since the bigger reorganization in 2016), partly to better benefit from the newly added DT competences. The process will continue throughout 2019; however, some actions have been decided. A first action is to co-locate employees with digital technology expertise, to ease collaboration and peer learning. A second action is to move the strategy focus of the innovation department to a separate strategy group, to concretize the focus of the innovation department on innovation, improvement, digitalization, and $\mathrm{R} \& \mathrm{D}$.

Third, it is not enough to build up DT competence. Once DT competences are in place, companies need to ensure that they keep the employees, and at the same time, continue to develop the competences. Thus, companies should continue to foster a digital culture, ensure that the necessary support and resources are in place, offer challenging tasks, and encourage a work environment characterized by continuous learning and experimentation. Appropriate leadership is also essential. Some argue that transformational leadership is particularly important in building a digital culture and motivating competence development in an organization [2]. This is important because without an appropriate digital culture, digital talents will not want to remain at the company [17].

\section{Conclusion}

A DT requires companies to rethink their competence base. In this paper, I provide insight from a Norwegian power grid company, to shed light on competence that is essential for established firms in their DTs. DT competence reflects a company's bundle of employee skills, knowledge, experience, etc., that are essential in a DT. In this study, employees' digital technology orientation, adoption, and expertise were found to be fundamental in GridCo's DT. Further, to better utilize digital technology and increased amounts of data, analytical skills, data management, and industry-specific analytics are essential. Finally, skills and knowledge within process improvement, digitalization, portfolio management, and agile project management were found to be important for GridCo to manage the DT, and embrace the changes that follow the DT.

This paper provides useful insight into competences essential in a DT, organized in a model (Figure 3) that can be useful for future studies, and for other companies in assessing their needs for DT competence. Further, the paper addresses how an established firm can attain DT competences, either through developing or acquiring relevant competences. As such, the research contributes to the DT literature in addressing one aspect of the broader question; how companies can succeed with digital transformation [19].

It is important to have in mind, however, that a DT is a continuous process, and DT competences are subject to change. Thus, it would be valuable to study changes in competence for a longer period, across different stages of the DT. As DT competence may differ depending on the specific needs of the organization and the particular industry in which the company operates [15], investigating DT competence in other established firms, and across industries, would also be valuable.

Figure 2 depicts the path GridCo has undergone in attaining DT competences, which can serve as inspiration for other companies in their DT, and a starting point for data analysis in future research. Combined with the practical implications outlined in section 7, I hope to be able to develop the figure further over time, to portray a more general path with potential approaches towards DT competence.

Finally, DT competence represents only one important element of a company's DT capability. Future studies should investigate other essential elements of DT capability as well, such as digital technology, leadership, and culture.

\section{References}

[1] Alam, K., Erdiaw-Kwasie, M. O., Shahiduzzaman, M., 
\& Ryan, B. (2018). "Assessing regional digital competence: Digital futures and strategic planning implications", Journal of Rural Studies, 60, pp. 60-69.

[2] Alos-Simo, L., Verdu-Jover, A. J., \& Gomez-Gras, J.M. (2017). "How transformational leadership facilitates ebusiness adoption", Industrial Management and Data Systems, 117(2), pp.382-397.

[3] Andriole, S. J. (2018). "Skills and Competences for Digital Transformation”, IT Professional, 20(6), pp. 78-81.

[4] Barney, J. (1991). "Firm Resources and Sustained Competitive Advantage", Journal of Management, 17, pp. 99-120.

[5] Bharadwaj, A. S. (2000). "A Resource-Based Perspective on Information Technology Capability and Firm Performance: An Empirical Investigation", MIS Quarterly, 24(1), pp. 169-196.

[6] Butschan, J., Heidenreich, S., Weber, B., \& Kraemer, T. (2018). "Tackling Hurdles to Digital Transformation - the Role of Competences for successful Industrial Internet of Things (IIoT) Implementation", International Journal of Innovation Management, 1950036, pp. 1-34.

[7] Creswell, J. W. (2012). Qualitative Inquiry and Research Design. Choosing Among Five Approaches (Third). Thousand Oaks, CA: SAGE publications.

[8] Dremel, C., Herterich, M. M., Wulf, J., Waizmann, J.C., \& Brenner, W. (2017). "How AUDI AG established big data analytics in its digital transformation", MIS Quarterly Executive, 16(2), pp. 81-100.

[9] Eden, R., Casey, V., Burton-Jones, A., \& Draheim, M. (2019). "Digital Transformation Requires Workforce Transformation", MIS Quarterly Executive, 18(1), pp. 114.

[10] Gao, S., Hakanen, E., Töytäri, P., \& Rajala, R. (2019). "Digital transformation in asset-intensive businesses: Lessons learned from the metals and mining industry", HICSS 2019 Proceedings, pp. 4927-4936.

[11] Grundke, R., Marcolin, L., Nguyen, T, L, B., Squicciarini, M. (2018). "Which skills for the digital era? Returns to skills analysis", OECD Science, Technology and Industry Working Papers 2018/09, pp. 1-36.

[12] Jorgensen, D. (1989). Participant Observation. Newbury Park, CA: SAGE publications.

[13] Kettunen, P., \& Laanti, M. (2017). "Future software organizations - agile goals and roles", European Journal of Futures Research, 5(1).

[14] Kettunen, P., \& Mäkitalo, N. (2019). "Future smart energy software houses", European Journal of Futures Research, 7(1).
[15] Khin, S., \& Ho, T. C. F. (2018). "Digital technology, digital capaility and organizational performance: A mediating role of digital innovation", International Journal of Innovation Science.

[16] Larsson-Lund, M., \& Nyman, A. (2018). "Occupational challenges in a digital society: A discussion inspiring occupational therapy to cross thresholds and embrace possibilities", Scandinavian Journal of Occupational Therapy.

[17] Lee, M.-X., Lee, Y.-C., \& Chou, C. J. (2017). "Essential Implications of the Digital Transformation in Industry 4.0", Journal of Scientific \& Industrial Research, 76 (August), pp. 465-467.

[18] Nwankpa, J. K., \& Roumani, Y. (2016). "IT Capability and Digital Transformation: A Firm Performance Perspective", ICIS 2016 Proceedings, pp. 1-16.

[19] Osmundsen, K., Iden, J., \& Bygstad, B. (2018). "Digital Transformation: Drivers, Success Factors, and Implications", MCIS 2018 Proceedings, Corfu, Greece.

[20] Peppard, J., \& Ward, J. (2004). "Beyond strategic information systems: Towards an IS capability", Journal of Strategic Information Systems, 13(2), pp. 167-194.

[21] Reis, J., Amorim, M., Melão, N., \& Matos, P. (2018). "Digital transformation: a literature review and guidelines for future research", World Conference on Information Systems and Technologies, pp. 411-421. Springer, Cham.

[22] Sebastian, I. M., Ross, J. W., Beath, C., Mocker, M., Moloney, K., \& Fonstad, N. O. (2017). "How Big Old Companies Navigate Digital Transformation", MIS Quarterly Executive, 16(3), pp. 197-213.

[23] Shahlaei, C., Rangraz, M., \& Stenmark, D. (2017). "Transformation of Competence - the Effects of Digitalization on Communicators' Work", ECIS 2017 Proceedings, pp. 195-209.

[24] Teece, D., Pisano, G., \& Shuen, A. (1997). "Dynamic Capabilities and Strategic Management", Strategic Management Journal, 18(7), pp. 509-533.

[25] Vial, G. (2019). "Understanding digital transformation: A review and a research agenda", Journal of Strategic Information Systems.

[26] Wade, M., \& Hulland, J. (2004). "The Resource-Based View and Information Systems Research: Review, Extension, and Suggestions for Future Research", MIS Quarterly, 28(1), pp. 107-142.

[27] Wiesböck, F., \& Hess, T. (2018). "Understanding the Capabilities for Digital Innovations from a Digital Technology Perspective", ArbeitsBericht 1/2018, LudwigMaximilians-Universität Munchen, pp. 1-20. 